INPLASY

PROTOCOL

To cite: Lin et al. Auricular therapy for tic disorder in children : a protocol for systematic review and metaanalysis. Inplasy protocol 202130074. doi:

10.37766/inplasy2021.3.0074

Received: 21 March 2021

Published: 21 March 2021

Corresponding author:

Zhengyang Shao

405316797@qq.com

Author Affiliation:

Hangzhou Red Cross Hospital

Support: No. $2021 Z A 108$.

Review Stage at time of this submission: Preliminary

searches.

Conflicts of interest:

None declared.

\section{Auricular therapy for tic disorder in children : a protocol for systematic review and meta-analysis}

Lin, TT1; Shao, ZY2; Lian, JL3; Zhang, $\mathrm{CH}^{4}$.

Review question / Objective: Among children and adolescents, Tic Disorder(TD) is a common neuropsychiatric disease which has a complex pathogenesis. In recent years, the prevalence of tic disorder is on the rise with the increase of life and study pressure, the penetration of electronic products and unhealthy diet. Because of its long treatment cycle, high recurrence and comorbidity with other neuropsychiatric disorders, tic disorder has a serious impact on patients' mental health and their society adaptiveness, which increases the burden on the family and society. As one of the important components of acupuncture, auricular therapy has been widely used for the treatment of tic disorder with the advantages of convenient operation and low cost. Nevertheless, there is still a lack of systematic review of auricular therapy for treating tic disorder in children.The purpose of this systematic review and meta-analysis is to evaluate the efficacy and safety of auricular therapy in the treatment of children with tic disorder.

INPLASY registration number: This protocol was registered with the International Platform of Registered Systematic Review and Meta-Analysis Protocols (INPLASY) on 21 March 2021 and was last updated on 21 March 2021 (registration number INPLASY202130074).

\section{INTRODUCTION}

Review question / Objective: Among children and adolescents, Tic Disorder(TD) is a common neuropsychiatric disease which has a complex pathogenesis. In recent years, the prevalence of tic disorder is on the rise with the increase of life and study pressure, the penetration of electronic products and unhealthy diet. Because of its long treatment cycle, high recurrence and comorbidity with other neuropsychiatric disorders, tic disorder has a serious impact on patients' mental health 
and their society adaptiveness, which increases the burden on the family and society. As one of the important components of acupuncture, auricular therapy has been widely used for the treatment of tic disorder with the advantages of convenient operation and low cost. Nevertheless, there is still a lack of systematic review of auricular therapy for treating tic disorder in children.The purpose of this systematic review and meta-analysis is to evaluate the efficacy and safety of auricular therapy in the treatment of children with tic disorder.

Condition being studied: Tic disorder (TD) is a neuropsychiatric developmental disorder characterized by multiple involuntary movements or abnormal vocalizations, which affecting between $0.5 \%$ and $0.8 \%$ of the world's population. The prevalence of chronic TD in school-age children in the United States reaches $0.3 \%$ to $5 \%$, while it is as high as $6.1 \%$ in China. The general clinical manifestations of tic disorder are vocal or motor tic, and it may turn to self-injurious behavior or obsessive-compulsive disorder in some severe cases. Without effective interventions, about $20 \%$ of child patients' tic symptoms may protract to their adulthood causing a seriously harm to the life quality and psychosocial function. So far the main treatment for TD is medication. While the behavioral and psychological therapy as adjuvant treatments. However, there is a greatly reduce of the efficacy and patient compliance, which is due to the side effects induced by combination of multiple drugs, the instability of the efficacy of non-pharmacological interventions (influenced by therapists' skill and experience in different region) and the high cost of treatment. Hence, it is necessary to find affordable, safe and effective treatments for TD. Auricular therapy is a traditional Chinese acupuncture therapy that prevents and treats diseases by stimulating the acupoints on the auricle. Currently, auricular therapy has been widely used to treat TD in children. However, due to the lack of clinical systematic evaluation and evidence-based medicine evidence. Therefore, it is necessary to conduct the first systematic review about the efficacy and safety of auricular therapy for TD in children.

\section{METHODS}

Search strategy: The search terms are combined with medical subject words (MESH) and text words. The key search terms are including tic disorder, transient tic disorder, chronic motor or vocal tic disorder, tourette syndrome, auricular acupoints, press-needle, auricular plaster therapy, auricular therapy, auricular acupressure therapy, auricular point pressure therapy, randomized controlled trial, controlled clinical trial, trials, randomized. Table 1 shows the search strategy for PubMed. Similar search strategy will be used for other online databases. Besides, the search strategy will be modified appropriately according to the differences between Chinese database and English database.

Participant or population: Patients in the participants must conform the clinical criteria for TD according to the Diagnostic and statistical manual of mental disorders (DSM-(労)). The patients' age limits 6 to16 years old. There is no restriction about nationality, race, gender, course of disease, lifestyle and family income level.

Intervention: The experimental group are mainly treated with auricular therapy, and there is no restriction that if only use auricular therapy or combined with other therapies, including traditional Chinese medicine, Chinese patent medicine, western medicine, massage, behavioral and psychological therapy, etc.

Comparator: The control group can be treated with traditional Chinese medicine, Chinese patent medicine, western medicine, massage, behavioral and psychological therapy or other combined therapies, but excluding auricular therapy.

Study designs to be included: All RCTs involving auricular therapy for the treatment of TD in children, whether if 
using blind method, will be included. The language of RCTs will not be restricted.

Eligibility criteria: We will include RCT to evaluate the effectiveness and safety of auricular therapy for the treatment of TD in children. The inclusion criteria are as follows: 1. The type of study is a randomized controlled trial.2. The study involved patients aged 6 to 16 years with clinically proven TD. The exclusion criteria are set as follows: 1.TD is a concomitant disease of other diseases; 2. Auricular therapy is used as an adjunct therapy; 3.Repeat publish; 4.No certain diagnostic criteria; 5 .The data is missing, incorrect, or unattainable.

Information sources: The following eight online databases including PubMed, Web of Science, Embase, Cochrane Library, China National Knowledge Infrastructure, WangFang Databases, Chinese Scientific Journal Database, Chinese Biomedical Literature Database will be searched from their establishment to March 2021. All RCTs will be selected and we will retrieve gray literature manually, including conference article and dissertation.

Main outcome(s): The primary outcomes are clinical efficacy and the score of YGTSS. 1. The clinical efficacy is evaluated based on the YGTSS score reduction rate, and the total effective rate is the sum of the cure rate, the apparent rate, and the effective rate; 2.YGTSS score is widely used to measure the severity of TD, which is a primary clinical outcome index to assess the severity of TD.

Additional outcome(s): The secondary outcomes included the following: 1.The score of Mini-CTIM; 2.The score of the GTS-QOL; 3.Adverse events.

Quality assessment / Risk of bias analysis: The quality of the studies will be assessed by using use the Cochrane risk assessment tool for assessing risk of bias, which assessed from the following aspects and items: selection (including random sequence and allocation concealment), implementation (including application of blind), measurement (blind evaluation of research findings), follow-up (integrity of outcome data), report (selectively reporting research findings) and other bias source. The judgment results of "low risk bias", "high risk bias" and "unclear" will be made for each item according to the bias risk assessment criteria. If any disagreement arises during the assessment process, this should be resolved through discussion with the third investigator.

Strategy of data synthesis: A meta-analysis of all included studies will be conducted using Review Manager5.3 provided by the Cochrane Collaboration. For dichotomous data, the effect index will use risk ratio (RR) or odds ratio (OR) with its $95 \%$ confidence interval $(95 \% \mathrm{Cl})$. For continuous variable, it will use mean deviation (MD) or standardized mean deviation (SMD) with its $95 \%$ CI.Firstly determining the heterogeneity. When the heterogeneity is small ( $P>0.1$ and $12<50 \%)$, using fixedeffects model. Otherwise, using the random effects model. We will compare the curative effect of treating TD patients between experimental group and control group by forest plot. Sensitivity analysis will be conducted in order to identify the source of heterogeneity according to the major factors that may lead to clinical heterogeneity.

Subgroup analysis: If the included studies are highly heterogeneous, we will conduct subgroup analysis based on the characteristics of the participants, intervention (such as simply auricular therapy, auricular therapy combined with different medications or psychotherapy) and treatment time.

Sensitivity analysis: In order to maintain the stability of the outcome indexes, we will use sensitivity analysis to assess the sample size, study design.

Language: English.

Country(ies) involved: China.

Keywords: auricular therapy; tic disorder; children; protocol; systematic review. 
Contributions of each author:

Author 1 - Tingting Lin.

Author 2 - Zhengyang Shao.

Author 3 - Junlan Lian.

Author 4 - Chunhui Zhang. 